Pacific Journal of Mathematics

LOCALIZATION IN THE CLASSIFICATION OF FLAT 


\title{
LOCALIZATION IN THE CLASSIFICATION OF FLAT MANIFOLDS
}

\section{Peter SymondS}

\begin{abstract}
Two compact flat Riemannian manifolds are called comparable if each one is a covering space of the other in such a way that the covering maps are affine and both the compositions of the covering maps increase distance locally by a constant factor. Considering comparability classes instead of affine-equivalence classes corresponds to localizing the algebra in calculations.
\end{abstract}

Introduction. This paper is concerned with compact flat Riemannian manifolds, i.e. smooth compact manifolds with a Riemannian connection for which the Levi-Civita connection is flat. These are all quotients of Euclidean space $\mathbf{R}^{n}$ by a group of isometries $\Gamma$ acting properly discontinuously. A continuous map between two such manifolds is called affine if it lifts to an affine map of $\mathbf{R}^{n}$. The rotational part of $\Gamma$, i.e. its image in $\mathrm{GL}\left(\mathbf{R}^{n}\right)$, is called the holonomy group of the manifold and is always finite. Charlap [4] showed that the affine-equivalence classes of these manifolds with given holonomy group $G$ correspond bijectively with the isomorphism classes of a category $E_{\mathbf{Z}}(G)$ defined in terms of the integral representations of $G$. For the purpose of calculation it is convenient to localize the integral representations to get a category $\hat{E}(G)$. This will be seen to correspond to the following geometric notion.

Definition. Two compact flat Riemannian manifolds $B_{1}, B_{2}$ are comparable if there exist affine covering maps $\theta_{1}: B_{1} \rightarrow B_{2}, \theta_{2}: B_{2} \rightarrow B_{1}$ such that $\theta_{1} \circ \theta_{2}$ and $\theta_{2} \circ \theta_{1}$ both increase distance locally by a factor $m$.

Section 1 covers the background material. In $\$ 2$ we shall look at the endomorphisms of these manifolds and in $\$ 3$ we shall prove the following.

THEOREM A. There is a natural bijection between the isomorphism classes of $\hat{E}(G)$ and the comparability classes of compact flat Riemannian manifolds with holonomy group $G$. 
Section 4 investigates the extent to which the cohomology of the manifold is an invariant of the comparability class and $\$ 5$ contains a calculation of the comparability classes when the holonomy group is the metacyclic group $D_{p q}, p, q$ primes.

1. Background. The main theorem on the structure of compact flat Riemannian manifolds is the following one $([\mathbf{1}],[3],[11])$.

THEOREM. If $\Gamma$ is the fundamental group of a compact flat Riemannian manifold of dimension $n$ then $\Gamma$ fits into a short exact sequence

$$
0 \rightarrow M \rightarrow \Gamma \rightarrow G \rightarrow 1
$$

in which

(i) $G$ is finite and

(ii) $M$ is free-Abelian of rank $n$. $G$ acts on $M$ by conjugation and

(iii) this action is faithful.

Any two embeddings of $\Gamma$ in $\operatorname{Isom}\left(\mathbf{R}^{n}\right)$, the group of isometries of $\mathbf{R}^{n}$, are conjugate in $\operatorname{Aff}\left(\mathbf{R}^{n}\right)$, the group of affine transformations of $\mathbf{R}^{n}$, and under such an embedding $M$ corresponds to the subgroup of pure translations and $G$ to the holonomy group.

Conversely, any abstract group which fits into an exact sequence with these properties can be realised as the fundamental group of a compact flat Riemannian manifold of dimension $n$, unique up to affine equivalence, and for each $n$ there are only finitely many such groups up to isomorphism. In fact any finite group can occur as the holonomy group $G$ of a compact flat Riemannian manifold for sufficiently large $n$ [1].

Thus the task of determining the compact flat Riemannian manifolds up to affine equivalence is equivalent to that of determining the groups $\Gamma$ which fit into a sequence (*) and satisfy conditions (i), (ii) and (iii). First of all, recall that if $R$ is a Dedekind ring and $G$ a finite group then a finitely-generated $R G$-module which is $R$-projective is called an $R G$-lattice. In the sequence (*) $M$ is a $\mathrm{Z} G$-lattice and the sequence is determined by an element $\alpha \in H^{2}(G ; M)$. We shall label the group $\Gamma$ and also the corresponding manifold (up to affine isomorphism) by $B(M, \alpha)$, or $B(G ; M, \alpha)$ if we wish to stress the role of $G$. There remains the question of whether different $M$ or $\alpha$ can lead to the same $\Gamma$. This was considered in [4]: define $\alpha \in H^{2}(G ; M)$ to be special if its restriction to each subgroup of prime order is non-zero. Then for any finite group $G$ we can define a category $E_{R}(G)$ to have as objects pairs $(M, \alpha)$ where $M$ is a 
faithful $R G$-lattice and $\alpha \in H^{2}(G ; M)$ is special, and as morphisms pairs $(f, A):(M, \alpha) \rightarrow(N, \beta)$ where $A$ is an automorphism of $G$ and to specify $f$ we define another $R G$-lattice $\hat{A}(N)$ to be like $N$ as an $R$-module but with a new action of $G$, denoted *,

$$
g^{*} m=A(g) m, \quad g \in G, m \in M
$$

$f$ is an $R G$-module homomorphism $M \rightarrow \hat{A}(N)$. There is an induced isomorphism $A_{*}: H^{2}(G ; M) \rightarrow H^{2}(G ; \hat{A}(N))$; we require $f_{*}(\alpha)=A_{*}(\beta)$.

TheOREM (Charlap). The map B defines a bijection between the isomorphism classes of $E_{\mathbf{z}}(G)$ and the affine-equivalence classes of compact flat Riemannian manifolds with holonomy group $G$.

This reduces the geometric problem of classifying the compact flat Riemannian manifolds with given holonomy group to an algebraic one involving Z $G$-lattices $M$ and $H^{2}(G ; M)$. Charlap [4] gave the solution for $G=C_{p}$, the cyclic group of order $p$, which is one of the few cases where all the $\mathbf{Z} G$-lattices are known.

The advantage of the comparability classes is that we only need to consider the genus of the $\mathbf{Z} G$-lattice $M$, where two $\mathbf{Z} G$-lattices $M, N$ are in the same genus if they are isomorphic when localized at any prime $p$ (we write $\left.M_{p} \cong N_{p}\right)$ or equivalently when completed at any prime $p\left(\hat{M}_{p} \cong\right.$ $\hat{N}_{p}$ ). See [7]. These are much easier to handle.

2. Construction of automorphisms. In order to decide whether two manifolds $B\left(G ; M, \alpha_{1}\right), B\left(G ; M, \alpha_{2}\right)$ are affinely equivalent we have to be able to construct automorphisms of $M$. We shall use the following proposition which applies to any ring $\Lambda$.

Proposition. Let $0 \rightarrow L \stackrel{r}{\rightarrow} M \stackrel{s}{\rightarrow} N \rightarrow 0$ be an exact sequence of $\Lambda$-modules determined by $\phi \in \operatorname{Ext}_{\Lambda}(N, L)$ and let $\alpha$ be an endomorphism of $L, \beta$ one of $N$ such that for some $\psi \in \operatorname{Ext}_{\Lambda}(N, L), \alpha_{*} \psi=\psi \beta_{*}=\phi$. Then there is an endomorphism $\gamma$ of $M$ which makes the following diagram commute.

$$
\begin{aligned}
0 \rightarrow & L \stackrel{r}{\rightarrow} M \stackrel{s}{\rightarrow} N \rightarrow 0 \\
& \alpha \downarrow \quad \gamma \downarrow \quad \beta \downarrow \\
0 \rightarrow & L \stackrel{r}{\rightarrow} M \stackrel{s}{\rightarrow} N \rightarrow 0
\end{aligned}
$$

Proof. Hilton and Stammbach [9] give an interpretation of the functor Ext in terms of extensions, not only for the modules but also for the morphisms. 
Let $\psi$ determine the extension $0 \rightarrow L \stackrel{r^{\prime}}{\rightarrow} M^{\prime} \stackrel{s^{\prime}}{\rightarrow} N \rightarrow 0$. By definition of $\alpha_{*}$ there is a commutative diagram

$$
\begin{aligned}
0 \rightarrow & \stackrel{\stackrel{r^{\prime}}{\rightarrow}}{\rightarrow} M^{\prime} \stackrel{s^{\prime}}{\rightarrow} N \rightarrow 0 \\
& \alpha \downarrow \quad \delta \downarrow \quad \text { id } \downarrow \\
0 \rightarrow L & \rightarrow M \rightarrow N \rightarrow 0
\end{aligned}
$$

in which the left-hand square is a push-out. By definition of $\beta_{*}$ there is a commutative diagram

$$
\begin{aligned}
0 \rightarrow & L \stackrel{r}{\rightarrow} M \stackrel{s}{\rightarrow} N \rightarrow 0 \\
& \text { id } \downarrow \quad \varepsilon \downarrow \quad \beta \downarrow \\
0 \rightarrow & L \stackrel{r^{\prime}}{\rightarrow} M^{\prime} \stackrel{s^{\prime}}{\rightarrow} N \rightarrow 0
\end{aligned}
$$

in which the right-hand square in a pull-back. Take $\gamma=\delta \varepsilon$.

Such an interpretation also works for $H^{2}(G ; M)$. In particular consider the endomorphism of $M$ which is just multiplication by a constant $m$. If $m$ is prime to $|G|$ then it does not affect $H^{2}(G ; M)$ so for any group $\Gamma$ which is an extension of $G$ by $M$ we get a monomorphism $\gamma$ satisfying

$$
\begin{aligned}
0 & \rightarrow M \rightarrow \Gamma \rightarrow G \rightarrow 1 \\
& m \downarrow \\
& \gamma \downarrow \text { id } \downarrow \\
0 & \rightarrow M \rightarrow \Gamma \rightarrow G \rightarrow 1 .
\end{aligned}
$$

If $M$ is a faithful $\mathbf{Z} G$-lattice then $\Gamma$ can be embedded in $\operatorname{Aff}\left(\mathbf{R}^{n}\right)$ and $\operatorname{im} \gamma$ must be conjugate to $\Gamma$ in $\operatorname{Aff}\left(\mathbf{R}^{n}\right)$ so if $\Gamma$ is torsion-free we get an affine map from the corresponding flat manifold to itself which evidently increases distances by a factor $m$.

Definition. An endomorphism of a flat manifold which increases all distances by a factor $m$ is called expanding of degree $m$.

THEOREM. A flat manifold has an expanding endomorphism of degree $m$ if and only if $m$ is prime to the order of the holonomy group.

Proof. We have shown the existence above. On the other hand any expanding map of degree $m$ leads to a diagram

$$
\begin{aligned}
0 \rightarrow M & \rightarrow \Gamma \rightarrow G \rightarrow 1 \\
m \phi \downarrow & \gamma \downarrow \\
0 \rightarrow M & \rightarrow \Gamma \rightarrow G \rightarrow 1
\end{aligned}
$$


with $A$ an automorphism of $G$ and $\phi$ an automorphism of $M$. The extension is determined by $\alpha \in H^{2}(G ; M)$ and the diagram implies $m \phi_{*} \alpha A_{*}^{-1}=\alpha$, but $\alpha$ is special so for any prime $p$ dividing $|G|$ let $C$ be a cyclic subgroup of order $p$; $\operatorname{res}_{C}^{G} \alpha \neq 0$ so $m \phi_{*} \operatorname{res}_{A^{-1}(C)} \alpha \neq 0$ so $p$ does not divide $m$, i.e. $m$ is prime to $|G|$.

REMARK. Epstein and Shub [8] construct expanding endomorphisms of degree $k|G|+1, k \in \mathbf{Z}, k \geq 0$.

3. Comparability. Recall that we defined two compact flat Riemannian manifolds $B_{1}, B_{2}$ to be comparable if there exist affine covering maps $\theta_{1}: B_{1} \rightarrow B_{2}, \theta_{2}: B_{2} \rightarrow B_{1}$ such that $\theta_{1} \circ \theta_{2}$ and $\theta_{2} \circ \theta_{1}$ are both expanding maps.

If $B_{1}=B\left(G_{1} ; M_{1}, \alpha_{1}\right), B_{2}=B\left(G_{2} ; M_{2}, \alpha_{2}\right)$ and $B_{1}$ and $B_{2}$ are comparable then we have a diagram

$$
\begin{aligned}
& 0 \rightarrow M_{1} \rightarrow \pi_{1}\left(B_{1}\right) \rightarrow G_{1} \rightarrow 1 \\
& \phi_{1} \downarrow \quad \downarrow \quad A_{1} \downarrow \\
& 0 \rightarrow M_{2} \rightarrow \pi_{1}\left(B_{2}\right) \rightarrow G_{2} \rightarrow 1 \\
& \phi_{2} \downarrow \quad \downarrow \quad A_{2} \downarrow \\
& 0 \rightarrow M_{1} \rightarrow \pi_{1}\left(B_{1}\right) \rightarrow G_{1} \rightarrow 1 .
\end{aligned}
$$

$\phi_{2} \circ \phi_{1}$ is multiplication by an integer $m$, prime to $p$, and $A_{2} \circ A_{1}$ is an automorphism of $G_{1}$. Also $A_{1} \circ A_{2}$ must be an automorphism of $G_{2}$ so $A_{1}$ and $A_{2}$ are isomorphisms.

$$
\phi_{1^{*}} \alpha_{1}=\alpha_{2} A_{2^{*}}, \quad \phi_{2^{*}} \alpha_{2}=\alpha_{1} A_{2^{*}}
$$

so

$$
m \alpha_{1}=\alpha_{1} A_{2^{*}} A_{1^{*}} .
$$

For any prime $p$ dividing $|G|$ let $C$ be a cyclic subgroup of order $p$, $\operatorname{res}_{C}^{G} \alpha_{1} \neq 0$ so $m \operatorname{res}_{\left(A_{2} A_{1}\right)^{-1} C}^{G} \alpha_{1} \neq 0$ so $p$ does not divide $m$, i.e. $m$ is prime to $|G|$.

Thus if we localize at any prime $p$ in $|G|$ then, in the category $E_{\mathbf{z}_{p}}(G)$, $\left(\phi_{2} \phi_{1, p}, A_{2} A_{1}\right)$ is an isomorphism as is $\left(\phi_{1} \phi_{2, p}, A_{1} A_{2}\right)$, so $\left(\phi_{1, p}, A_{1}\right)$ is mono and epi and hence $\phi_{1, p}$ is an isomorphism of $M_{1, p}$ with $M_{2, p}$ if we identify $G_{1}$ with $G_{2}$ using $A_{1} . M_{1}$ and $M_{2}$ must be in the same genus under this identification and $\phi_{1}$ induces an isomorphism

$$
\phi_{1^{*}}: H^{2}\left(G_{1} ; M_{1}\right) \rightarrow H^{2}\left(G_{1} ; M_{2}\right) \text { with } \phi_{1^{*}} \alpha_{1}=\alpha_{2} \text {. }
$$

Definition. Suppose $B_{1}=B\left(G_{1} ; M_{1}, \alpha_{1}\right), B_{2}=B\left(G_{2} ; M_{2}, \alpha_{2}\right)$. We shall say that $B_{1}$ and $B_{2}$ are algebraically comparable if and only if for some identification $G_{1} \cong G_{2}=G$, say, $M_{1}$ is in the same genus as $M_{2}$ and for each prime $p$ dividing $|G|$ there is an isomorphism $\psi_{p}: \hat{M}_{1, p} \rightarrow \hat{M}_{2, p}$ such that if $\alpha_{i, p}$ is the image of $\alpha_{i}$ in $H^{2}\left(G ; \hat{M}_{i, p}\right)$ then $\psi_{p^{*}} \alpha_{1, p}=\alpha_{2, p}$. 
We shall need the following lemma which is based on one of Roiter [cf. 7, p. 645], see also [5].

LEMMA A. Let $M$ and $N$ be $\mathbf{Z} G$-lattices in the same genus, so for each $p$ dividing $|G|$ there are isomorphisms $\psi_{p}: \hat{M}_{p} \rightarrow \hat{N}_{p}$. Then there is a homomorphism $\phi: M \rightarrow N$ such that $\hat{\phi}_{p^{*}}=\psi_{p^{*}}: H^{*}\left(G ; \hat{M}_{p}\right) \rightarrow H^{*}\left(G ; \hat{N}_{p}\right)$ and there is an exact sequence

$$
0 \rightarrow M \stackrel{\phi}{\rightarrow} N \rightarrow T \rightarrow 0
$$

with $T$ finite of order prime to $|G|$.

Proof. $\hat{\mathbf{Z}}_{p} \otimes \operatorname{Hom}_{\mathbf{z} G}(M, N) \cong \operatorname{Hom}_{\hat{\mathbf{z}}_{p} G}\left(\hat{M}_{p}, \hat{N}_{p}\right) . \operatorname{Hom}_{\mathbf{Z} G}(M, N)$ is densely embedded in $\operatorname{Hom}_{\hat{\mathbf{z}}_{p} G}\left(\hat{M}_{p}, \hat{N}_{p}\right)$ under the $p$-adic topology so for each $p$ dividing $|G|$ we may choose $u_{p} \in \operatorname{Hom}(M, N)$ with $\hat{u}_{p} \equiv \psi_{p} \bmod p^{r}$ where $r$ is large enough that $p^{r}$ does not divide $|G|$. By the Chinese Remainder Theorem choose integers $\alpha_{p}$ such that

$$
\alpha_{p} \equiv 1 \bmod p^{r}, \quad \alpha_{p} \equiv 0 \bmod p^{r^{\prime}}, \quad p^{\prime} \neq p
$$

where $p^{\prime}$ varies the over all primes dividing $|G|$ except $p$, and $p^{\prime r^{\prime}}+|G|$. Then $\phi=\sum_{p \| G \mid} \alpha_{p} u_{p}$ has the correct effect on cohomology since $\phi_{p} \equiv$ $u_{p} \bmod p^{r}$ and $H^{*}(G ;-)_{p}$ is annihiliated by $p^{r}$. Similarly we can get $\theta \in \operatorname{Hom}(N, M)$ and

$$
\hat{\theta}_{p} \hat{\phi}_{p} \equiv 1 \bmod p^{r}, \quad \hat{\phi}_{p} \hat{\theta}_{p}=1 \bmod p^{r} .
$$

By Nakayama's lemma, $\hat{\theta}_{p} \hat{\phi}_{p}$ is onto $\hat{M}_{p}$ so $\hat{\theta}_{p} \hat{\phi}_{p}$ must be an automorphism of $\hat{M}_{p}$ and in particular $\operatorname{ker} \hat{\phi}_{p}=0$. Hence $(\operatorname{ker} \phi)_{p}=0$ and since $\operatorname{ker} \phi$ is torsion-free, $\operatorname{ker} \phi=0$. Let $T=\operatorname{coker} \phi$, so

$$
0 \rightarrow M \stackrel{\phi}{\rightarrow} N \rightarrow T \rightarrow 0 .
$$

Since $\hat{\phi}_{p}$ is an isomorphism, $\hat{T}_{p}=0$, so $T$ is finite and $|T|$ is prime to $|G|$.

Now if we let $m=|T|, m N \subset \phi(M)$, so we can define $\phi^{\prime}: N \rightarrow M$ by $\phi^{\prime}(n)=\phi^{-1}(m n), n \in N$. On cohomology $\phi_{*}$ is iso and $\phi_{*}^{\prime} \phi_{*}=m$ is iso so $\phi_{*}^{\prime}$ is iso. By the proposition of $\S 2$ and the discussion afterwards, $\phi$ and $\phi^{\prime}$ lead to covering maps $\theta: B_{1} \rightarrow B_{2}, \theta^{\prime}: B_{2} \rightarrow B_{1}$ which show that $B_{1}$ and $B_{2}$ are comparable. We have shown:

THEOREM B. Algebraic comparability is equivalent to comparability for flat manifolds. 
It is now clear how to define the category $\hat{E}(G)$ in order to make Theorem A valid. The objects are the same as those of $E_{\mathbf{z}}(G)$ but the morphisms must be those occurring in the definition of algebraically comparable. That is, a morphism $(M, \alpha) \rightarrow(N, \beta)$ is now a pair $\left(\left\{f_{p}\right\}, A\right)$ where $A$ is an automorphism of $G$ and for each $p$ which divides $G$ there is a $\hat{\mathbf{Z}} G$-module automorphism $f_{p}: \hat{M}_{p} \rightarrow \hat{A}\left(\hat{N}_{p}\right)$ such that $f_{p^{*}}\left(\alpha_{p}\right)=A_{*}\left(\beta_{p}\right)$.

REMARKS. (a) Since there are exist non-isomorphic $\mathbf{Z} G$-lattices in the same genus, for example when $G$ is cyclic of order 23 , the construction yields examples of manifolds which cover each other in a non-trivial way. Charlap [4] has examples which become affinely equivalent after taking the product with a circle.

(b) It is perhaps interesting to see what happens if we weaken the condition in the definition of comparable that the compositions should be expanding. A lot of the integral representation theory is lost: for example let $B(G ; M, \alpha)$ be a flat manifold and let $S, T$ be two $\mathbf{Z} G$-lattices which are isomorphic as $\mathbf{Q} G$-spaces, but not even in the same genus. Then $B(G ; M \oplus S, \alpha \oplus 0)$ and $B(G ; M \oplus T, \alpha \oplus 0)$ both cover each other (since $S$ is isomorphic to a submodule of $T$ of finite index and vice versa).

4. The cohomology of a flat manifold. It is interesting to see how the cohomology of a flat manifold $B(G ; M, \alpha)$ depends on $M$ and $\alpha$. We shall calculate $H^{* 9 B}$ ) using the spectral sequence of the extension $0 \rightarrow M$ $\rightarrow \pi_{1}(B) \rightarrow G \rightarrow 1$, (since $B$ is an Eilenberg-MacLane space $\left.\left.K\left(\pi_{1}(B), 1\right)\right)\right)$. For coefficients in a ring $R$,

$$
E_{2}^{i j}(R)=H^{i}\left(G ; H^{j}(M ; R)\right)=H^{i}\left(G ; \Lambda^{j} H^{1}(M ; R)\right) .
$$

Proposition. The Betti numbers of $B(G ; M, \alpha)$ depend only on $M$.

Proof. $E_{2}^{i j}(\mathbf{Q})=0$ unless $i=0$.

$$
H_{j}(B ; \mathbf{Q}) \cong E_{2}^{0 j}(\mathbf{Q}) \cong\left(\Lambda^{j}(M \otimes \mathbf{Q})\right)^{G} .
$$

Proposition. The $E_{2}$ terms of the spectral sequence (with any coefficients) depend only on the genus of $M$.

Proof. The torsion-free part is taken care of as above. As for the torsion, $H^{1}(M ; R) \cong \operatorname{Hom}(M, R)$ and $H^{i}(G ; N)_{p} \cong H^{i}\left(G ; N_{p}\right)$ and

$$
\operatorname{Hom}(M, R) \otimes \mathbf{Z}_{p} \cong \operatorname{Hom}\left(M_{p}, R \otimes \mathbf{Z}_{p}\right) \text {. }
$$


THEOREM. If two flat manifolds $B_{1}, B_{2}$ are comparable then the cohomology groups $H^{*}\left(B_{1} ; \mathbf{Z}\right), H^{*}\left(B_{2} ; \mathbf{Z}\right)$ are isomorphic. If $R$ is a subring of $\mathbf{Q}$ with all primes not in a finite set inverted or $R$ is a field then there is an affine map $f: B_{1} \rightarrow B_{2}$ which induces an isomorphism of rings $f^{*}$ : $H^{*}\left(B_{2} ; R\right) \cong H^{*}\left(B_{1} ; R\right)$.

Proof. Suppose $R \subset \mathbf{Q}$ and all primes not in $|B|$ are invertible in $R$. We shall show that $\theta^{*}: H^{*}\left(B_{2} ; R\right) \rightarrow H^{*}\left(B_{1} ; R\right)$ is an isomorphism when $\theta: B_{1} \rightarrow B_{2}$ is a covering map constructed in the proof that algebraically comparable implies comparable. Let $\phi: M_{1} \rightarrow M_{2}$ be the induced map on $\mathbf{Z} G$-lattices; it is sufficient to show that this gives an isomorphism $\phi^{*}$ : $H^{1}\left(M_{2} ; R\right) \rightarrow H^{1}\left(M_{1} ; R\right)$ (since $H^{j}\left(M_{i} ; R\right) \cong \Lambda^{j} H^{1}\left(M_{i} ; R\right)$ ). But, by construction, coker $\phi$ is finite of order prime to $|G|$ and so from the exact sequence

$$
\begin{gathered}
0 \rightarrow \operatorname{Hom}(\operatorname{coker} \phi, R) \rightarrow \operatorname{Hom}\left(M_{2}, R\right) \rightarrow \operatorname{Hom}\left(M_{1}, R\right) \rightarrow \operatorname{Ext}(\operatorname{coker} \phi, R) \\
\text { " }
\end{gathered}
$$

we see that this is the case $\left(H^{1}(M ; R) \cong \operatorname{Hom}(M, R)\right)$. For other $R$ we must examine the proof of Lemma A to see that coker $\phi$ can be made coprime to an additional finite set of primes.

The only torsion that can occur in $H^{*}(B ;-)$ is at primes in $|G|$ since the only $E_{2}^{* *}$ terms which are not annihilated by $|G|$ are the $E_{2}^{0 *}$ and there are no differentials with image in $E^{0 *}$. Thus we can deduce the result for $R=\mathbf{Z}$ from the case with the primes not in $|G|$ inverted.

REMARKS. (a) A version of this theorem for $G$ of prime order was proved by Charlap and Vasquez [5], who also calculate the groups $H^{*}(B ; \mathbf{Z})$ in this case.

(b) It is not known whether the rings $H^{*}(B ; \mathbf{Z})$ must be isomorphic.

5. Metacyclic groups. Let $D_{p q}$ be a metacyclic group of order $p q$, with $p, q$ distinct primes and $q$ dividing $p-1$ :

$$
\begin{gathered}
1 \rightarrow C_{p} \rightarrow D_{p q} \rightarrow C_{q} \rightarrow 1, \\
D_{p q}=g p\left\langle x, y \mid x^{p}=y^{q}=1, y x y^{-1}=x^{r}\right\rangle,
\end{gathered}
$$

where $r$ is a primitive $q$ th root of $1 \bmod p$. We shall find the comparability classes of flat manifolds with holonomy group isomorphic to $D_{p q}$. The genera of the indecomposable $D_{p q}$-lattices were determined by $\mathrm{Pu}[\mathbf{1 0}]$ and are given in [7, pp. 747-751], whose description we follow here. 
Let $R=\mathbf{Z}[\zeta$ ], where $\zeta$ is a primitive $p$ th root of 1 . Define an automorphism $\sigma$ of $R$ over $\mathbf{Z}$ by $\sigma(\zeta)=\zeta^{r}$ and let $D_{p q}$ act on $R$ by

$$
\begin{aligned}
& x r=\zeta r, \\
& y r=\sigma(R), \quad r \in R .
\end{aligned}
$$

Thus $R$ is a $\mathbf{Z} G$-lattice and so is the ideal $P=(1-\zeta) R$ or any power of it, $P^{i}$. There are also the indecomposable lattices for the factor $C_{q}$, in particular $\mathbf{Z}, \mathbf{Z} H$ and its augmentation ideal $S$, where $H=\langle y\rangle$.

If we localize at $q$ the indecomposable $\mathbf{Z}_{q} D_{p q}$-lattices are as follows (we shall calculate the cohomology groups later, but include them here for convenience).

$$
\begin{array}{cc}
\frac{\mathbf{Z}_{q} D_{p q} \text {-lattice } M}{\mathbf{Z}_{q}} & \frac{H^{2}\left(D_{p q} ; M\right)}{\mathbf{Z} / q} \\
S_{q} & 0 \\
\mathbf{Z}_{q} H & 0 \\
R_{q} & 0
\end{array}
$$

Alternatively if we complete at $p$ the indecomposable lattices are

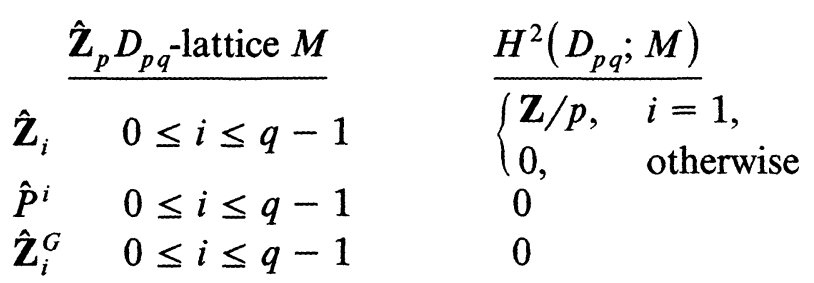

$\hat{\mathbf{Z}}_{i}$ is a copy of $\hat{\mathbf{Z}}_{p}$ on which $x$ acts as 1 and $y$ acts as $\theta^{\mathrm{i}}$, where $\theta$ is the primitive $q$ th root of 1 in $\hat{\mathbf{Z}}_{p}$ with $\theta \equiv r \bmod \left(p \mathbf{Z}_{p}\right)$. $\hat{\mathbf{Z}}_{i}^{G}$ is induced from $\hat{\mathbf{Z}}_{i}$ restricted to $H$ and is also the unique non-split extension of $\hat{\mathbf{Z}}_{i}$ by $\hat{P}^{i+1}$

$$
0 \rightarrow \hat{P}^{i+1}+\hat{\mathbf{Z}}_{i}^{G} \rightarrow \hat{\mathbf{Z}}_{i} \rightarrow 0 .
$$

LemMa. (See [2].) Let $N$ be a normal subgroup of $G$ with $|N|$ prime to $|G / N|$. Then

$$
H^{r}(G ; M) \cong H^{r}\left(G / N ; M^{N}\right) \oplus H^{r}(N ; M)^{G / N} .
$$

Proof. Use the spectral sequence for $1 \rightarrow N \rightarrow G \rightarrow G / N \rightarrow 1$.

REMARK. If the action of $N$ on $M$ is trivial, so $H^{2}(N ; M) \cong$ $H^{1}(N ; \mathbf{Q} \otimes M / M) \cong \operatorname{Hom}(N, \mathbf{Q} \otimes M / M)$, then the action of $G$ on $H^{2}(N ; M)$ is given by

$$
\left({ }^{g} f\right)(x)=g f\left(g^{-1} x g\right), \quad f \in \operatorname{Hom}(N, \mathbf{Q} \otimes M / M), \quad x \in N, g \in G
$$


The cohomology can now be calculated using the formula for the cohomology of a cyclic group. Observe that if $M$ is $\mathbf{Z}_{q} D_{p q}$-lattice then $H^{2}\left(D_{p q} ; M\right)$ has only $q$-torsion so $H^{2}\left(D_{p q} ; M\right) \cong H^{2}\left(D_{p q} / C_{p} ; M^{C_{p}}\right)$. Hence the results for $\mathbf{Z}_{q}, S_{q}$ and $\mathbf{Z}_{q} H$. Also, $R_{q}^{C_{p}}=0$.

Over $\hat{\mathbf{Z}}_{p}, H^{2}\left(D_{p q} ; M\right) \cong H^{2}\left(C_{p} ; M\right)^{H}$.

$$
H^{2}\left(C_{p} ; P^{i}\right)=0 \text {. }
$$

$H^{2}\left(D_{p q} ; \hat{\mathbf{Z}}_{i}^{G}\right) \cong H^{2}\left(H ; \hat{\mathbf{Z}}_{i}\right) \cong 0$ since $\mathbf{Z}_{i}^{G}$ is induced from $\mathbf{Z}_{i}$.

$H^{2}\left(C_{p} ; \hat{\mathbf{Z}}_{i}\right) \cong \operatorname{Hom}\left(C_{p},\left(\hat{\mathbf{Q}}_{p} \otimes_{\hat{\mathbf{z}}_{p}} \hat{\mathbf{Z}}_{i}\right) / \hat{\mathbf{Z}}_{\imath}\right) \cong \operatorname{Hom}\left(C_{p}, \hat{\mathbf{Z}}_{i} / p \hat{\mathbf{Z}}_{i}\right)$.

Let $f \in \operatorname{Hom}\left(C_{p}, \hat{\mathbf{Z}}_{i} / p \hat{\mathbf{Z}}_{i}\right)$; then if $f={ }^{y} f$,

$$
\begin{aligned}
f(x) & =y f\left(y^{-1} x y\right)=\theta^{i} f\left(x^{s}\right) \quad \text { where } s r \equiv 1 \bmod p \\
& =s \theta^{i} f(x),
\end{aligned}
$$

so $i=1$.

Representatives of the $2^{q}+2^{q-1}+q+2$ indecomposable genera of the $D_{p q}$-lattices are as follows.

$\begin{array}{cccc}\begin{array}{c}\text { lattice } M \\ P^{i}, \begin{array}{c}0 \leq i \leq q-1 \\ \mathbf{Z}\end{array}\end{array} & \frac{M_{q}}{R_{q}} & \frac{\hat{M}_{p}}{\hat{P}_{l}} & \frac{H^{2}\left(D_{p q} ; M\right)}{0} \\ S & \mathbf{Z}_{q} & \hat{\mathbf{Z}}_{0} & \mathbf{Z} / q \\ H & S_{q} & \sum_{l=1}^{q-1} \hat{\mathbf{Z}}_{\mathrm{i}} & \mathbf{Z} / p \\ X_{T} & \mathbf{Z}_{q} H & \sum_{i=0}^{q-1} \hat{\mathbf{Z}}_{i} & \mathbf{Z} / p \\ Y_{T} & S_{q}+R_{q}^{|T|} & \sum_{i \in T} \hat{\mathbf{Z}}_{l-1}^{G}+\sum_{l \notin T \cup\{1\}} \hat{\mathbf{Z}}_{l-1} & \begin{cases}\mathbf{Z} / p & \text { if } 2 \notin T(\bmod q) \\ 0 & \text { otherwise }\end{cases} \\ V & \mathbf{Z}_{q} H+R_{q}^{|T|} & \sum_{i \in T} \hat{\mathbf{Z}}_{l-1}^{G}+\sum_{l \notin T} \hat{\mathbf{Z}}_{l-1} & \begin{cases}\mathbf{Z} / p & \text { if } 2 \notin T(\bmod q) \\ 0 & \text { otherwise } \\ \mathbf{Z}_{0}^{G} & \mathbf{Z} / q\end{cases} \end{array}$

where $T$ is any non-empty subset of $\{0,1, \ldots, q-1\}$ except that to form $X_{T}$ we cannot have $1 \in T$.

The cohomology groups are as given since $H^{*}(G ; M)_{p} \cong H^{*}\left(G ; M_{p}\right)$ $\cong H^{*}\left(G ; \hat{M}_{p}\right)$ for any prime $p$.

$H^{2}\left(D_{p q} ; M\right)$ has a special point if and only if it has both $p$-torsion and $q$-torsion since $\operatorname{cor}_{H}^{G} \operatorname{res}_{H}^{G}=|G: H| x$. In any case the comparability class depends only on the genus since all the $p$-torsion in $H^{2}\left(D_{p q} ; M\right)$ comes from $\hat{\mathbf{Z}}_{p}$ and all the $q$-torsion from the irreducible $\mathbf{Z}_{q}$ and it is easy to construct an automorphism of these which takes any non-zero element of $H^{2}$ to any other. 
However the automorphisms of the group permute the $\hat{\mathbf{Z}}_{i}, i \neq 0$, and hence also the $P^{i}, i \neq 1$, in order to preserve sequence $(\dagger)$. This permutes the sets $T$ by changing the elements not equal to 1 according to $\alpha \mapsto$ $(\alpha-1)^{r}+1 \bmod q$ for some $r$ depending on the automorphism, and any $r$ prime to $q$ is possible. Note that it acts in the same way on each copy of $T$.

THEOREM C. The comparability classes of flat manifolds with holonomy group isomorphic to $D_{p q}$ are in one-to-one correspondence with the equivalence classes of the genera of faithful special $D_{p q}$-lattices under the relation on $T$ described above.

\section{REFERENCES}

[1] L. Auslander and M. Kuranishi, On the holonomy group of locally Euclidean spaces, Ann. Math., 65 (1957), 411-415.

[2] A. Babakhanian, Cohomological Methods in Group Theory, Dekker, New York, 1972.

[3] L. Bieberbach, Über die Bewegunsgruppen der Euklidische Räume, Math. Ann., 70 (1910), 287-336 and 72 (1912), 400-412.

[4] L. Charlap, Compact flat Riemannian manifolds I, Ann. Math., 81 (1965), 15-30.

[5] L. Charlap and A. Vasquez, Compact flat Riemannian manifolds II, Amer. J. Math., (1965), 551-563.

[6] P. Cobb, Manifolds with holonomy group $\mathbf{Z}_{2} \oplus \mathbf{Z}_{2}$ and first Betti number zero, J. Differential Geom., 10 (1975), 221-224.

[7] C. W. Curtis and I. Reiner, Methods of Representation Theory, John Wiley, New York, 1981.

[8] D. Epstein and M. Shub, Expanding endomorphisms of flat manifolds, Topology, 7 (1968), 139-141.

[9] P. Hilton and U. Stammbach, A Course in Homological Algebra, Springer-Verlag, Berlin, Heidelberg, New York, 1971.

[10] L. C. Pu, Integral representations of non-Abelian groups of order pq, Michigan Math. J., 12 91985), 231-246.

[11] H. Zassenhaus, Beweis eines Satzes über diskrete Gruppen, Abh. Math. Semin. Univ. Hamburg, 21 (1938), 289-312.

Received October 25, 1985 and in revised form July 1, 1986.

The OHIo State University

Columbus, OH 43210 



\section{PACIFIC JOURNAL OF MATHEMATICS EDITORS}

\author{
V. S. VARADARAJAN \\ (Managing Editor) \\ University of California \\ Los Angeles, CA 90024 \\ HeRbert Clemens \\ University of Utah \\ Salt Lake City, UT 84112 \\ R. FINN \\ Stanford University \\ Stanford, CA 94305
}

\author{
HERMANN FLASCHKA \\ University of Arizona \\ Tucson, AZ 85721 \\ RAMESH A. GANGOLLI \\ University of Washington \\ Seattle, WA 98195 \\ VAUGHAN F. R. JONES \\ University of California \\ Berkeley, CA 94720 \\ ROBION KIRBY \\ University of California \\ Berkeley, CA 94720
}

\author{
C. C. MOORE \\ University of California \\ Berkeley, CA 94720 \\ H. SAMELSON \\ Stanford University \\ Stanford, CA 94305 \\ HAROLD STARK \\ University of California, San Diego \\ La Jolla, CA 92093
}

\section{ASSOCIATE EDITORS}
R. Arens
E. F. BECKENBACH
B. H. NEUMANN
F. WOLF
K. YosHIDA (1906-1982)

\section{SUPPORTING INSTITUTIONS}

UNIVERSITY OF ARIZONA
UNIVERSITY OF BRITISH COLUMBIA
CALIFORNIA INSTITUTE OF TECHN
UNIVERSITY OF CALIFORNIA
MONTANA STATE UNIVERSITY
UNIVERSITY OF NEVADA, RENO
NEW MEXICO STATE UNIVERSITY
OREGON STATE UNIVERSITY

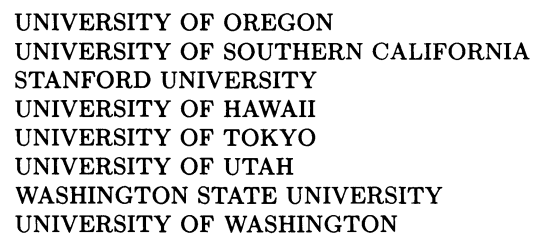

The Supporting Institutions listed above contribute to the cost of publication of this Journal, but they are not owners or publishers and have no responsibility for its content or policies.

Mathematical papers intended for publication in the Pacific Journal of Mathematics should be in typed form or offset-reproduced (not dittoed), double spaced with large margins. Please do not use built up fractions in the text of the manuscript. However, you may use them in the displayed equations. Underline Greek letters in red, German in green, and script in blue. The first paragraph must be capable of being used separately as a synopsis of the entire paper. In particular it should contain no bibliographic references. Please propose a heading for the odd numbered pages of less than 35 characters. Manuscripts, in triplicate, may be sent to any one of the editors. Please classify according to the scheme of Math. Reviews, Index to Vol. 39. Supply name and address of author to whom proofs should be sent. All other communications should be addressed to the managing editor, or Elaine Barth, University of California, Los Angeles, California 90024.

There are page-charges associated with articles appearing in the Pacific Journal of Mathematics. These charges are expected to be paid by the author's University, Government Agency or Company. If the author or authors do not have access to such Institutional support these charges are waived. Single authors will receive 50 free reprints; joint authors will receive a total of 100 free reprints. Additional copies may be obtained at cost in multiples of 50 .

The Pacific Journal of Mathematics is issued monthly as of January 1966. Regular subscription rate: $\$ 190.00$ a year (5 Vols., 10 issues). Special rate: $\$ 95.00$ a year to individual members of supporting institutions.

Subscriptions, orders for numbers issued in the last three calendar years, and changes of address should be sent to Pacific Journal of Mathematics, P.O. Box 969, Carmel Valley, CA 93924, U.S.A. Old back numbers obtainable from Kraus Periodicals Co., Route 100, Millwood, NY 10546.

The Pacific Journal of Mathematics at P.O. Box 969, Carmel Valley, CA 93924 (ISSN 0030-8730) publishes 5 volumes per year. Application to mail at Second-class postage rates is pending at Carmel Valley, California, and additional mailing offices. Postmaster: send address changes to Pacific Journal of Mathematics, P.O. Box 969, Carmel Valley, CA 93924.

PUBLISHED BY PACIFIC JOURNAL OF MATHEMATICS, A NON-PROFIT CORPORATION Copyright (C) 1987 by Pacific Journal of Mathematics 


\section{Pacific Journal of Mathematics \\ Vol. 127, No. $2 \quad$ February, 1987}

Richard Martin Aron and Robert Henry Lohman, A geometric function determined by extreme points of the unit ball of a normed space .... . . 209

Kari Astala and M. S. Ramanujan, $(s)$-nuclear sets and operators $\ldots \ldots \ldots 233$ Earl Robert Berkson, Thomas Alastair Gillespie and Paul Scott Muhly, Analyticity and spectral decompositions of $L^{p}$ for compact abelian

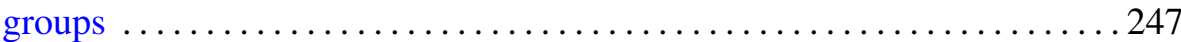

Ronald P. Brown, Real closures of fields at orderings of higher level . . . . . 261

Ronald P. Brown, The behavior of chains of orderings under field

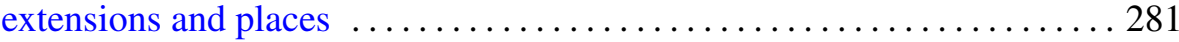

Rodney Graham Downey, Subsets of hypersimple sets .............. 299

Manfred Droste and Saharon Shelah, On the universality of systems of

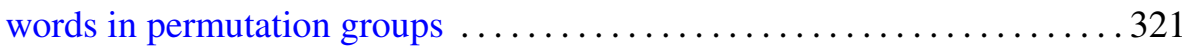

Hidenori Fujiwara, Représentations monomiales des groupes de Lie

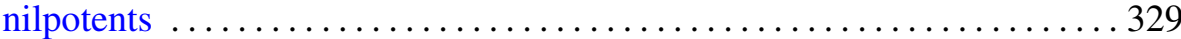

Rupert Lasser, Convolution semigroups on hypergroups $\ldots \ldots \ldots \ldots \ldots 35$

Marcus Marlene Marsh, $u$-mappings on trees $\ldots \ldots \ldots \ldots \ldots \ldots \ldots \ldots \ldots \ldots$

Peter Andrew Symonds, Localization in the classification of flat

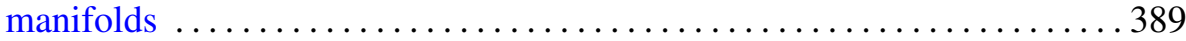

